Research Article

\title{
Comprehensive Evaluation of Urban Road Network Resilience Facing Earthquakes
}

\author{
Lin Gao $\mathbb{D},{ }^{1}$ Mingzhen Wang $\mathbb{D},{ }^{1}$ Anshuang Liu $\mathbb{i}{ }^{2},{ }^{2}$ and Huafeng Gong $\mathbb{D}^{2}$ \\ ${ }^{1}$ College of Civil Engineering, Chongqing University of Arts and Sciences, Chongqing, China \\ ${ }^{2}$ T. Y. Lin International Engineering Consulting (China) Co., Ltd., Chongqing, China \\ Correspondence should be addressed to Mingzhen Wang; wmz917@126.com
}

Received 9 November 2020; Revised 7 March 2021; Accepted 20 April 2021; Published 3 May 2021

Academic Editor: Erik Cuevas

Copyright (C) 2021 Lin Gao et al. This is an open access article distributed under the Creative Commons Attribution License, which permits unrestricted use, distribution, and reproduction in any medium, provided the original work is properly cited.

The road network's transport capacity and traffic function will be directly reduced if urban roads are damaged by earthquakes. To effectively improve the resistance and recovery ability of urban road networks facing earthquake disasters, the establishment of an aseismic resilience evaluation method for the urban road network is the research goal. This paper's novelty introduces the concept of engineering resilience into the aseismic performance evaluation of urban road networks. It reveals the internal influence principle of nodes and independent pathways on the aseismic resilience of the network. This paper's significant contribution is to establish a reasonable and comprehensive urban road network aseismic resilience evaluation method. This method can realize the calculation of the aseismic resilience for the existing network, reconstruction network, and new network and propose the optimization, transformation, and layout for the network. The MATLAB program for the whole process calculation of aseismic resilience is developed. The overall network's aseismic resilience is obtained by the sum of the product of the node importance and the average number of the reliable independent pathways.

\section{Introduction}

With the rapid development of urbanization, cities today are increasingly dependent on water, electricity, gas, transportation, communications, and other crucial urban infrastructures. Lifeline infrastructures are the lifeline of the city. How to ensure that the infrastructure can quickly recover its function when subjected to external shock or damage effect is an important issue facing urban development and the key issue to build a resilient city. Among them, the performance of urban road networks is essential. Under the rapid growth of the economy, information, and logistics, the road network is no longer merely a transfer of persons or goods and extends to all aspects of the whole social and economic life. The urban road system is a multiple-direction and grade separation network composing of multinodes and multilinks. The performance of node and link determines the overall network performance. Earthquake is one of the most severe natural disasters, and it is a potential major threat to urban infrastructure and economic development. The network performance reduction, even partial or total network paralysis caused by seismic actions that directly led to bridges, tunnels, slope structure damage, or buildings damages along the street, indirectly led to road section blocked. How to reasonably evaluate and improve urban road networks' aseismic resilience is needed to solve.

The influencing factors and management frameworks of disaster resilience for the city and community are studied. Complex urban system resilience to face natural disasters was learned by Katarina Rus in 2018 [1]. Urban systems should be analyzed as a whole resilience, taking into account the physical components such as buildings, infrastructures, open spaces, and social components such as community and the dynamic interactions between them. An integrated framework for multisector infrastructure asset management, including sharing information, risk management, resilience, and sustainability, was proposed by Yang [2].

Scholars gave the specific definition and connotation of resilience for urban infrastructure networks. In 2019, six urban critical infrastructure networks, namely, water, 
drainage, gas, transportation, electric, and communication, were focused. The resilience definitions, hazard categories, methodologies, and enhanced measures for each network were analyzed in detail [3]. In the same year, the literature review method was adopted to obtain and summarize the transportation system's resilience concept, which was divided into topological metrics, attribute-based metrics, and performance-based metrics. The resilience measurement methods, including optimization model, topology model, simulation model, probabilistic model, fuzzy logic model, and data-driven model, had been proposed. The large-scale transportation networks, social and economic, and resilience system of interdependent systems in certain scenes should be considered in the future development of transportation system resilience [4]. The evaluation indicators and methods were established based on the definition of resilience. The network performance was quantized based on network service function by many researchers, such as flow capacity [5], connectivity [6-9], and travel time [8]. But these indicators were mainly used to measure network performance under normal service conditions. They could not effectively reflect the network's resilience and sensitivity under natural and human-made hazards with high destructive and low occurrence probability, such as earthquakes, floods, and terrorist attacks. Peeta [10] used network connections and postdisaster traversing cost between the multiple origin nodes to destination nodes for predisaster investment decisions. Morlok and Chang [11] proposed capacity flexibility to reflect the adaptive capacity of traffic patterns change when the transportation system was under the impact of natural disasters. Chang and Nojima [12] introduced the concept of network coverage and traffic accessibility when networks recovered after disasters. Ip and Wang [13] thought that the redundancy of pathways between all origin nodes to destination nodes could be used to measure the resilience of transportation network. The above performance indicators had their advantages when the network performances were quantified under hazardous conditions. Shang et al. analyzed the robustness of urban road networks from topological and operational perspectives based on the complex network theory [14].

The resilience evaluation index to measure road networks' performance had been proposed based on network reliability and connectivity in 2016. The independent pathways between two nodes that do not share any road section were selected as the basis for resilience evaluation [15-17]. The important degree of a node was expressed as the relative distance to emergency resources, and only road sections, including bridge structures, were considered in the network. Different periods including predisaster mitigation, emergency response, and long-term disaster recovery were analyzed and modeled in detail. The Monte Carlo algorithm was used to calculate the connected state between network nodes. The method proposed by Zhang for evaluating network resilience with independent pathways had been widely adopted in recent years [18]. The resilience-based bilevel programming model was established for both deterministic and stochastic cases to determine the transportation network recovery strategy during the emergency recovery phase. An algorithm that integrated GA for PMSP and the Frank-Wolfe algorithm for UE was designed. The method considered the interaction between the system resilience maximization and the postdisaster user routechoice behavior. The optimization strategy of the transportation network recovery during the emergency recovery phase under uncertainty was investigated in the paper [19].

The resilience evaluation system of the transportation network was applied in many ways. As early as 2001, the REDARS shortened by risks from earthquake damage to roadway systems was developed by National Center for Earthquake Engineering Research. The system could simulate the entire performance of the road network to fight the earthquake. The models of seismology, geology, engineering science, economics, and so on were focused on estimating direct losses and indirect losses of systems affected by disasters. But the model relied on detailed seismic observation data. The REDARS had been demonstrated and applied to the road network at Tennessee Shelby County and Los Angeles, California, USA. In 2018, the resilience of large and complex metro networks was assessed by quantitatively analyzing, including vulnerability and recovery rapidity. The network efficiency indicated the network connectivity performance. It was found that the vulnerability of the network depended not only on the node degree of the disrupted station but also on its contribution to the whole network connectivity [20]. The resilience properties of bridges considered critical links in highway networks were evaluated based on postdisaster direct and indirect losses. The indirect losses, including connectivity losses and time prolongations, were proposed by Davide Forcellini [21]. The urban road network's concepts and metrics under snow weather were analyzed based on a resilient city's perspective. The mathematical model of the restoration for snow and ice weather road network was established. The emergency supplies layout problem and optimization problem of snow removal operation were solved, and the corresponding inspires solver algorithm was designed [18]. The urban road network's infrastructure service capacity in the established model was defined as the average speed on all independent pathways, which refers to Zhang's resilience model [15].

Wang [22] proposed the evacuation method of urban road network resilience under extreme snow events. The mathematical model for road network recovery under extreme weather and the snow removal resource locationallocation problem was established. And the proposed model and algorithm verified the effectiveness by a real case. At the same time, the interactional resilience between the two interdependent infrastructure systems was also analyzed. In 2019, the interdependent civil infrastructure system resilience was analyzed based on a physics-based synthesis framework. The framework consisted of four major components: setting resilience analysis boundary, establishing an infrastructure resilience management lifecycle, defining the physics-based modeling of infrastructure system functions, and designing the interfaces between interdependent infrastructure systems. The resilience analysis of the two interconnected infrastructure systems named stormwater drainage system and road transport system was integrated 
with the physics-based vulnerability analysis methods by using the ArcGIS platform [23]. The interdependent systems of transportation and electric power systems were considered based on network flow analysis methods. The dynamic traffic assignment algorithm, rather than the static algorithm, was used to capture more realistic traffic dynamics in congested urban roadway networks. Traffic demands, uncertain disruptions, repair costs, and mitigation actions were also considered in the problem formulation [24]. The resilience of community infrastructure systems was defined as the joint probability of robustness and rapidity and quantified using Bayesian networks. The framework was applied for resilience evaluation of building, transportation, water, and electric power infrastructure systems in Seaside, Oregon. The seismic ground shaking and tsunami inundation hazards corresponding to different return periods were also considered simultaneously [25].

To sum up, the resilience network focusing on performance is mainly studied in the existing research of transportation network resilience. In contrast, the actual network resilience should be a complex synthesis integrated into physics, performance, economy, and society. The road network's static properties, including topological form and road section reliability, need to be considered in the resilience analysis of urban road networks facing earthquake disasters. Simultaneously, the dynamic properties, including the emergency resource distributions, traveler's itinerary preference, should also be emphatically considered. The resilience for a local or global network of interest can be calculated and compared by the established resilience model. Therefore, this paper aims to address seismic safety for the urban road network based on the concept of "quickly recoverability from disasters." The seismic safety evaluation system of the urban road network is established to determine the best mitigation strategy and effectively guarantee and improve seismic safety, which can provide a reference method for the aseismic resilience performance evaluation of the urban road network.

\section{Map Generation of the Urban Road Network}

Building a network map is a critical step in the research of complex urban road networks. The geographically visible road intersection and the route can be abstracted into recognizable symbols and forms in network analysis. Therefore, the generation of the urban road network model is the basis for the network characteristics analysis.

\subsection{Method Selection of the Urban Network Generation.} The urban road transportation network is an essential part of the transportation infrastructure, and it has similarities with other modes in terms of transportation functionality. Both classical method and dual method are commonly used in the modeling of the transportation network.

2.1.1. Modeling by the Classical Method. According to intuitive cognition, the network routes are as links, and the intersections or joints between links in the network are as nodes, which is called classical method modeling. This method is the most straightforward mapping of the road network, and all geospatial information of road transportation is retained. The classical method modeling is widely used in urban geography and transportation planning.

2.1.2. Modeling by the Dual Method. In contrast to the classical method, the routes in the network are taken for nodes, and the intersections or joints between nodes in the network are as links, which is called dual method modeling. The dual method is divided into direct duality, road name duality, and continuation duality. The dual method is complicated than the classic method, but it can reflect the road network's more profound attributes. Different abstract ways of the above two methods are used when mapping road networks, and the characteristics of the two are shown in Table 1.

Table 1 shows that the classic method is worse than the dual method in the profound analysis effect for the network. Still, the former than the latter has more advantages in reflecting the network structure directly. The ordinary node attributes and structural attributes of the urban road network are analyzed in this research, and the requirements for modeling methods are relatively low. Therefore, the classical method is selected to build the urban road network model in this paper.

2.2. Model Generating Step of the Urban Road Network. The modeling of the urban road network is constructed according to the classical method. The intersections or roundabouts simplify as the nodes, and the routes between nodes simplify as links. The detailed procedures of modeling generation are as follows:

(1) Let $V$ be the set of nodes for the urban road network; the network node set including $n$ nodes is $V=\{1,2$, $\ldots \ldots, i, \ldots ., n\}$.

(2) Let $E$ be the set of links for the urban road network, the link between node $i$ and node $j$ is named edge $e_{i j}$, and the set is $E=\left\{e_{11}, e_{12}, \ldots \ldots, e_{i j}, \ldots \ldots, e_{n n}\right\}$. When there is no link between node $i$ and node $j, e_{i j}$ takes the value 0 .

Therefore, a theoretical model of urban road network $G=(V, E)$ is built using complex network theory.

According to the theoretical model, the whole urban road networks are visualized. The first step of network visualization is needed to collect specific road information, and then links and nodes are transformed into the network data. The detailed procedures of the simplified network diagram are as follows:

(1) Collecting the basic information of the analyzed network by Google maps, Baidu maps, or other platforms

(2) Screening the nodes in the network and selecting the critical intersection, roundabouts, and interchanges as the road network node 
TABLE 1: The characteristics of the modeling method for the transportation network.

\begin{tabular}{lcc}
\hline Method & & Characteristic \\
\hline $\begin{array}{l}\text { Classical } \\
\text { method }\end{array}$ & $\begin{array}{c}\text { Simple, intuitive, geographic features of the road network which can be reflected, a large number of } \\
\text { nodes, uniform edge distribution, poor profound analysis effect of network } \\
\text { Intuitive, simple, large network complexity }\end{array}$ \\
$\begin{array}{l}\text { Dual } \\
\text { method }\end{array}$ & $\begin{array}{c}\text { Road name dual } \\
\text { method } \\
\text { Continuation dual } \\
\text { method }\end{array}$ & Historical influence and more subjective factors \\
& Less network complexity, small correlation of geographic features, but large calculation amount \\
\hline
\end{tabular}

(3) Screening the links in the network and selecting urban expressway and major road as the link of the road network

(4) Using AutoCAD software to draw a visual model of the road network and transforming road model information into the matrix data

(5) Calculating and analyzing the road network data using the prepared MATLAB program

After the above procedures, the aseismic resilience performance of the road network is obtained ultimately.

After generating the abstract model of the urban road network, the network comprises nodes and links. Therefore, the node feature, the network structure, and the link reliability directly affect the road network's resilience.

\section{Node Feature and Node Importance}

3.1. Node Feature. Node feature is divided into four aspects considering both the administrative hierarchy and importance degree. The four aspects are degree centrality, betweenness centrality, accessibility, and resource occupy, respectively.

3.1.1. Degree Centrality. The key node as a hub in the network topology can be represented as its degree centrality. The degree $d c_{i}$ of node $i$ is defined as the number of links directly connected to node $I$ and the number of edges connected to node $i$ in the simplified network diagram. The more links connected to a node, the more important the node is in the network topology. The degree $d c_{i}$ of each node in the network is obtained firstly, and then degree centrality $D C_{i}$ of each node is got after normalizations by the formula of $D C_{i}=d c_{i} / \max \left\{d c_{i}\right\}$.

3.1.2. Betweenness Centrality. Node importance in the transmission process of the information flow is the node's degree located in an "intermediate position" in the other pathways. It can be represented as the betweenness centrality of the node. Betweenness centrality is the probability that the network node is located in the shortest pathway between any two nodes. That is, to what extent is a node located in an "intermediate position" between any other nodes. The betweenness centrality $b c_{i}$ of each node in the network is obtained firstly. The betweenness centrality $B C_{i}$ of each node is got after normalizations by the formula of $B C_{i}=b c_{i} / \max$ $\left\{b c_{i}\right\}$.
3.1.3. Accessibility. The accessibility from a node to other nodes in the network can be defined as the number of alternative passing ways between nodes. The more the alternative passing ways, the more the number of all pathways and the higher the accessibility. The parameter $a_{i}$ represents the number of all pathways between nodes $i$ and the other $(n-1)$ nodes. The accessibility $A_{i}$ is obtained after normalizations by the formula of $A_{i}=a_{i} / \max \left\{a_{i}\right\}$.

3.1.4. Resource Occupy. The criticality of nodes on the administrative level and the emergency resource supply can be expressed by the relative distance from the nearest administrative center, fire center, or rescue center, and so on. The shortest distance from node $i$ to the urban community emergency response facilities reflects the relative importance of resource availability for node $i$ in postdisaster emergency response work. Let $V$ denote the set of all network nodes. Let $E$ represent the nodes' set, including administrative center, fire center, or rescue center. Let $N$ be the subtraction of the $V$ set and $E$ set, and the nodes in the $N$ set do not include emergency response resources. $L_{S_{i, j}}$ represents the shortest pathway length between node $i$ and node $j$, where $j \in E$ and $i \in N$. The node $j$ may be only selected one of the administrative centers, fire centers, or rescue centers based on the actual situation. The resource availability index $E R_{i}$ listed in equation (1) represents the relative distance from node $i$ to the nearest emergency resource, which shows the importance degree of node $i$ on the aspect of emergency resource supply. For the case of the minimum value for $j$ is $1, L_{S_{i, j}}$ is 1 ; the influence of some distance from the node to emergency resource, the $E R_{i}$ value is multiplied by 0.9 on the result from

$$
E R_{i}=\left\{\begin{array}{l}
\frac{1}{\min \left\{L_{S_{i, j}} \mid j \in E\right\}}, \quad i \in N, \\
1, \quad i \in E .
\end{array}\right.
$$

3.2. Node Importance. The hub degree, center degree, accessibility, and resources occupy degree are considered in node importance named $I_{i}$, which is calculated according to

$$
I_{i}=\alpha_{1} D C_{i}+\alpha_{2} B C_{i}+\alpha_{3} A_{i}+\alpha_{4} E R_{i},
$$

where $\alpha_{1}, \alpha_{2}, \alpha_{3}$, and $\alpha_{4}$ are the weighting factor of $D C_{i}, B C_{i}$, $A_{i}$, and $E R_{i}$, respectively. 
The relationship between the four weighting factors is $\alpha_{1}+\alpha_{2}+\alpha_{3}+\alpha_{4}=1$. The network's working status is divided into three: the daily operation phase, the emergency recovery phase, and the comprehensive recovery phase. The daily operation phase refers to the network's functional state before the disaster, at which time, the nodes and links have high reliability. The emergency recovery phase refers to the golden rescue time from the moment of the disaster to 72 hours after the disaster. After the disaster, the comprehensive recovery phase refers to the period from 72 hours after the disaster to one month. After the recovery of this period, the link's function in the network is restored, and it is close to the daily operation phase after one month. Network resilience performances of different phases have different requirements and metrics, so the values of weighting factors are different in the model. When the connectivity performance of the daily operation phase of the network is stressed, $\alpha_{1}, \alpha_{2}$, and $\alpha_{3}$ have preferred a larger value. When the emergency rescue capability of the emergency recovery phase is emphasized, $\alpha_{3}$ and $\alpha_{4}$ are taken a larger value. The suggested values of node weighting factor $\alpha$ for each phase are listed in Table 2. The larger the value of the weighting factor for a particular item, the greater its impact on network resilience performance. According to the actual situation, the weighting factors can be assigned different values by urban transport managers or government decision-makers, which embodies openness and applicability of the evaluation model for network resilience performance.

\section{Network Structure and Pathway Importance}

4.1. Network Structure. The structure morphology of the road network is usually manifested in a topology structure, which reflects the physical characteristics of the road network. The network connectivity, travel time, traffic capacity, and so on are dependent on the performance and the number of the pathways between nodes. According to the different connection statuses, the pathways between nodes can be divided into the shortest pathways, all pathways, and independent pathways.

(1) The shortest pathway is the shortest distance connecting between two nodes. The shortest pathways for postdisaster emergency rescue, evacuation, and transfer have great significance. The network reliability regarded as the shortest pathway has been extensively studied. Generally speaking, the number of the shortest pathways between two nodes is limited, and the minimum quantity is one.

(2) All possible connections between two nodes compose all pathways, which are divided into the shortest pathway, the shorter pathway, the longer pathway, and the longest path according to the pathway length division. Most of all pathways share some of the same links. When the share link's reliability decreases, the reliability of the pathways, including shared links, is directly affected. The more the number of pathways between two nodes, the more the passing ways between two nodes and the greater the reliability
TABLE 2: The suggested values of weighting factor $\alpha$ for each phase.

\begin{tabular}{lcccc}
\hline Phase & $\alpha_{1}$ & $\alpha_{2}$ & $\alpha_{3}$ & $\alpha_{4}$ \\
\hline Daily operation phase & 0.30 & 0.30 & 0.30 & 0.10 \\
Emergency recovery phase & 0.10 & 0.10 & 0.20 & 0.60 \\
Comprehensive recovery phase & 0.20 & 0.20 & 0.30 & 0.30 \\
\hline
\end{tabular}

between two nodes. The number of all pathways between nodes in the complex network is typically huge. The number of all pathways between node $i$ and the other $(n-1)$ nodes can reflect the position of node $i$ in the network hierarchy to a certain extent. When the number of all pathways between nodes $i$ and the other $(n-1)$ nodes is small, node $i$ locates near the network's center position. Conversely, node $i$ locates near the edge of the network.

(3) The independent pathway refers to the pathway without any shared link between node $i$ and node $j$. The identification process of the independent pathway is as follows: all pathways database between node $i$ and node $j$ is calculated and obtained $\longrightarrow$ the shortest pathway $S_{i j}$ between two nodes is found $\longrightarrow$ remove the pathways shared with the shortest pathway from the all pathways database, and then the intermediate pathways database is obtained $\longrightarrow$ remove the pathways with shared links in the intermediate pathways database according to the shortest path principle $\longrightarrow$ the independent pathways database including one shortest pathway between the node $i$ and node $j$ is got. In terms of the quantity of all pathways, the number of independent pathways is generally limited, the independent pathways are without any shared link, and the reliability of each independent pathway is relatively independent. The greater the number of independent pathways between node $i$ and node $j$, the weaker the influence by the shared links and the more stable the number of effective connectivity pathways between nodes. Because the premise of the number of independent pathways is small and relatively stable, the reliability, average daily traffic volume, length, and travel time of independent pathways are easy to obtain, so the efficiency of data processing is higher.

According to the above analyses, the specific relationships of three aspects for network structure are shown in Figure 1. All independent pathways, including one shortest pathway, are considered to be the core of resilience performance for road networks resistant to disasters. Since the types and quantities of the links for a certain independent pathway are not the same, resulting in not the same reliability, traffic, travel time, and length, each independent pathway's comprehensive importance is different.

4.1.1. Reliability. The resilience performance of the network is directly determined by the reliability of the pathway. The $k$ th independent pathway named $P_{k}(i, j)$ between nodes $i$ 


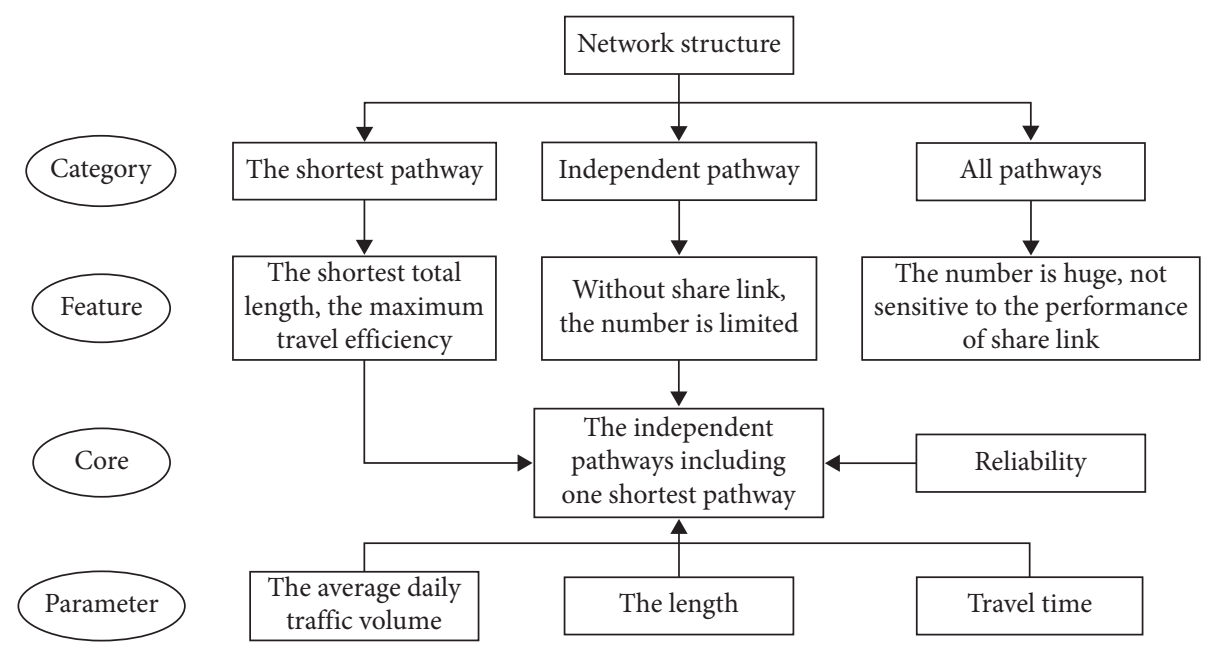

FIGURE 1: Characteristics of network structure.

and $j$ is connected by each link $l$ in series, so the reliability $R_{P_{k}(i, j)}$ of the independent pathway $P_{k}(i, j)$ is the continued production of the reliabilities $r_{l}$ of links $l$ included in $P_{k}(i, j)$; that is, $R_{P_{k}(i, j)}=\prod_{\forall l \in P_{k}(i, j)} r_{l}$.

4.1.2. Average Daily Traffic. The pathway's average daily traffic reflects this pathway's relative influence on personal life activities and local economic development. The pathway between the origin-destination (O-D) pairs with a shorter length and a greater traffic flow has a big contribution to the network function, which should be taken into account the network's resilience performance. The average daily traffic volume can be estimated from the monitoring data of actual traffic flow or traffic assignment models. It is considered that the minimum average daily traffic volume of each link in an independent pathway can determine the traffic volume of this pathway. Let vl represent the traffic volume of the link 1 , and let $v_{P_{k}(i, j)}$ represent the minimum average daily traffic volume of links. It means the equation of $v_{P_{k}(i, j)}=\min \left\{v_{l} \mid l \in P_{k}(i, j)\right\}$. The average daily traffic of the independent pathway is normalized by using the equation of $V_{P_{k}(i, j)}=\left(v_{P_{k}(i, j)} / \max \left(v_{P_{k}(i, j)}\right)\right)$, and the index average daily traffic is obtained.

4.1.3. Length. The total length of the pathway can portray the ease or complexity of that a node arrives through the network at the other nodes in the complex network, which is the sum of the length $l_{l}$ for each link $l$ included in the pathway. The total length of the independent pathway $P_{k}(i, j)$ is calculated by the equation of $l_{P_{k}(i, j)}=\sum_{l \in P_{k}(i, j)} l_{l}$. The shorter the total length of the pathway, the higher the travel efficiency and the more favors the travelers. Let $l_{\min }(i, j)$ represent the minimum length of all independent pathways between nodes $i$ and $j$. The length of the independent pathway is normalized by using the equation of $L_{P_{k}(i, j)}=\left(1 / l_{P_{k}(i, j)}\right) \cdot l_{\min }(i, j)$, and the length index named $L_{P_{k}(i, j)}$ is obtained.
4.1.4. Travel Time. The travel time of a link is related to the length and the traffic volume, but also the width of the link. In order to directly reflect traffic jams and travel efficiency, the index of travel time is selected as an evaluation criterion. Let $s_{l}$ represent the average speed of the link $l$ in a certain period, which can be acquired from big traffic data or obtained by the various map application in real-time time. Let $t_{l}$ be the average travel time for vehicles of the link $l$, so the relationship of $t_{l}=l_{l} / s_{l}$ exists. The total travel time of the $k$ th independent pathway is $t_{P_{k}(i, j)}=\sum_{\forall l \in P_{k}(i, j)} t_{l}$. Let $t_{\min }(i, j)$ be the minimum travel time of all total travel times of independent pathways between nodes $i$ and $j$. The travel time of the independent pathway is normalized by using the equation of $T_{P_{k}(i, j)}=\left(1 / t_{P_{k}(i, j)}\right) \cdot t_{\min }(i, j)$, and the index of travel time named $T_{P_{k}(i, j)}$ is obtained.

4.2. Pathway Importance. Pathway importance named $g_{P_{k}(i, j)}$ is related to the average daily traffic, length, and travel time. Pathway importance reflects that this pathway impacts personal life activities, local economic development, and postdisaster relief, which is calculated according to

$$
g_{P_{k}(i, j)}=\beta_{1} V_{P_{k}(i, j)}+\beta_{2} L_{P_{k}(i, j)}+\beta_{3} T_{P_{k}(i, j)},
$$

where $\beta_{1}, \beta_{2}$, and $\beta_{3}$ are the weighting factor of $V_{P_{k}(i, j)}, L_{P_{k}(i, j)}$, and $T_{P_{k}(i, j)}$, respectively.

The relationship between the three weighting factors is $\beta_{1}+\beta_{2}+\beta_{3}=1$. The way to assume values of the weighting factor for the independent pathway is the same as to node weighting factor, and the values of weighting factors can be assigned by urban transport managers or government decision-makers according to the actual situation. When the network usage function is stressed, $\beta_{1}$ and $\beta_{2}$ have preferred a larger value. When the network traffic efficiency is emphasized, $\beta_{2}$ and $\beta_{3}$ are taken a larger value. When the postearthquake quick emergency repair and resource configuration are emphasized, $\beta_{2}$ is chosen a larger value. The suggested values of weighting factor $\beta$ for independent pathways in each phase are listed in Table 3. 
TABLE 3: The suggested values of weighting factor $\beta$ for each phase.

\begin{tabular}{lccc}
\hline Phase & $\beta_{1}$ & $\beta_{2}$ & $\beta_{3}$ \\
\hline Daily operation phase & 0.20 & 0.30 & 0.50 \\
Emergency recovery phase & 0.10 & 0.50 & 0.40 \\
Comprehensive recovery phase & 0.5 & 0.25 & 0.25 \\
\hline
\end{tabular}

\section{Link Reliability}

The differences in topography and hydrology in the cities lead to different types of links in urban road networks. The complicated resilience calculation model is taken into account herein. The types of link include bridges, tunnels, slopes, and ordinary road sections, which is referred to as the network components. The reliabilities of the network components are the securities of links in the network. The component performance indexes refer to the reliability of bridges, the reliability of tunnels, the reliability of the slopes, and the reliability of the ordinary road sections [26].

(1) Bridge reliability: from the view of seismic performance for the bridge, the bridge damage index is selected as the indicator of bridge reliability refers to [27-29].

(2) Tunnel reliability: the tunnel damage index is selected as the indicator of bridge reliability, which can be calculated by the seismic damage evaluation method of tunnels proposed by Lin [30].

(3) Slope reliability: many road sections in the mountainous city are built down the mountains. The secondary disaster such as slope failure and landslide caused by the earthquake is the main cause of road sections damages in the mountainous city based on historical damage experience. The slope stability index is selected as an indicator reflecting the slope reliability, which can be calculated by the slope damage prediction method proposed by Wang [31].

(4) Reliability of ordinary road section: the road structure itself is damaged to some extent when suffered earthquake disaster. The major failure modes of the ordinary road section have cracks, bumps, spraying of the pavement and roadbed collapse, and so on. A strong earthquake usually does not cause damage to the road structure itself, according to earthquake damage investigations, so the damage of the road structure itself is not considered in this paper. But when a strong earthquake occurs, a lot of debris from buildings of collapse or serious damage along the street has a major influence on the traffic capacity of the road section. Therefore, the amount of debris is the decisive factor for the connectivity of the ordinary road section. The reliability of the road section proposed by Du [32] can be used to calculate the ordinary road section's reliability.

\section{Comprehensive Evaluation Model of Aseismic Resilience}

Comprehensive Evaluation Method of resilience performance is built based on node-link diagram from Section 2 and influence factor from Section 3 and Section 4 together. Each influence factor is normalized in order to horizontal compare factors. Node importance, pathway importance, and comprehensive evaluation model are described sequentially in this section.

The road network's resilience performance metric referred to as $R P M$ is calculated by equations (4) and (5). Aseismic resilience of road networks is related to node importance, pathway importance, and pathway reliability. The core of aseismic resilience calculation is node importance and the average number of reliable independent pathways. The product of the importance and reliability of all independent pathways between node $i$ and node $j$ is obtained to represent the number of the reliable independent pathways between node $i$ and node $j$. The ratio of the number of the reliable independent paths from node $i$ to all other nodes to $(n-1)$ represents the average number of the reliable independent pathways between node $i$ and other $(n-1)$ nodes in the network. The average number of the reliable independent pathways multiplied by the importance of node $i$ is the aseismic resilience of node $i$, and the resilience performance metric called RPM of the whole network is obtained by summing the aseismic resilience of all nodes. The meaning and role of each parameter in the equations are shown in Table 4.

$$
\mathrm{RPM}=\sum_{i=1}^{n} I_{i} P_{i}
$$

$P_{i}=\frac{1}{n-1} \sum_{j=1, j \neq i}^{n} \sum_{k=1}^{K(i, j)} g_{P_{k}(i, j)} \cdot R_{P_{k}(i, j)}$.

The resilience performance of an existing network with topological structure and functional status is evaluated. For the network with less resilience, the ways of changing network topology, enhancing the importance of key nodes, or the reliability of links can be employed to improve the network's resilience performance. The resilience performances of changed networks are calculated repeatedly, then the degrees of network resilience improvement are compared, and optimal risk control measures are identified based on the above analyses.

\section{Calculation and Method Comparison of Instance Network}

The established comprehensive evaluation method of network aseismic resilience performance in Section 4 is employed to calculate the instance network in the literature of Zhang [15], and both results are compared. The resilience calculation method proposed by Zhang [15] is simply known as the Zhang model for short in below. 
TABLE 4: The meaning and role of parameters in the equations.

\begin{tabular}{|c|c|}
\hline Parameter & Meaning and role \\
\hline$R P M$ & The resilience performance metric of the road network \\
\hline$n$ & The number of all nodes in the network \\
\hline$i$ & A node in the network, $V$ is a set of nodes, $i \in V[1, \ldots, \mathrm{n}], i$ is usually as the origin \\
\hline & A node in the network, $V$ is a set of nodes, $j \in V[1, \ldots, \mathrm{n}], j$ is usually as the destination \\
\hline$P_{i}$ & $\begin{array}{c}\text { The average number of reliable independent pathways considering pathway importance between node } i \text { and any other }(n-1) \\
\text { nodes in the network }\end{array}$ \\
\hline$K(i, j)$ & The total number of independent pathways between node $i$ and node $j$ \\
\hline$k$ & Counting the number of independent pathways \\
\hline $\begin{array}{l}g_{P_{k}(i, j)} \\
P_{k}(i, j)\end{array}$ & The $k$ th independent pathway named $P_{k}(i, j)$ connected by each link $l$ in series between node $i$ and node $j$ \\
\hline
\end{tabular}

7.1. Calculation Methods and Features of Zhang Model. The network's resilience performance is divided into two aspects of node and pathway in the Zhang model. The node aspect mainly considers the ability to supply emergency resources. The value of the node importance is the reciprocal of the shortest distance from the nearest emergency response facilities, which reflects the importance of node $i$ in the postdisaster emergency work. The pathway aspect is considered the amount, reliability, and importance for the independent pathway without any shared link. Only the road sections including bridges are taken into account, and the reliability of the bridge is the reliability of the road section. The importance of independent pathways is determined based on the average daily traffic and the length in two ways. The shorter the length, the larger the average daily traffic and the more important the independent pathway. Finally, the overall network's resilience is the product of the node weighting factor and the average number of reliable independent pathways. The whole thought of the Zhang model is shown in Figure 2.

7.2. Resilience Calculation of Instance Network. The established evaluation method in Section 4 is used to calculate the resilience of the instance network, and then the results that came from the present model and Zhang model [15] are compared. The instance network is shown in Figure 3 [15], wherein node 9 and node 17 include the emergency response facilities. The data of reliability, average daily traffic, and restoration cost for each road section in the network can be found in this paper's supplemental file.

The aseismic resilience at some particular states of the network in the literature of Zhang [15] is calculated by using the model proposed in this paper. The more detailed calculation processes and results can be seen in the supplemental file of this paper. The resilience metric of postdisaster is 3.73 using the proposed model, which means that there are 3.73 reliable independent pathways existing between any origin and a destination in the instance network. Disaster reduction decisions, including before and after disasters in enhancing the aseismic resilience effects, are analyzed and compared, and the results are shown in Tables 5 and 6 . In the tables, S1 represents the added link between nodes 19 and 27, S2 represents the added link between nodes 22 and 26, S3 represents the added link between nodes 27 and 29, S4 represents the added link between nodes 9 and 15, and S5 represents the added link between nodes 8 and 21. Before the disaster, the reliability of all added links is 0.999 , and the length of each added link is 1 . The average daily traffic of the added S1, S2, S3, S4 and S5 links are assumed 2800, 2800, 2900, 2500, and 1700, respectively. Only the links are added before the disaster, and other conditions are the same as in Section 5.2.

There are two main means of promoting network resilience to resist seismic hazard: improving the reliability of key links and changing the network topology. Four kinds of maintenance plans under available total restoration costs are provided, and the reliability of the link resumes to 0.999 after repairing. There are a total of 37 links in the original network shown in Figure 3. When 7 links that occupied 19\% of the total links are repaired by using 28 units cost, the network resilience increases by $16.9 \%$ compared to 3.73 after the disaster; when $43 \%$ of the total links are repaired by using 65 units cost, the network resilience increases of $52.0 \%$. When $73 \%$ of the total links are repaired by using 105 units cost, the network resilience increases by $91.7 \%$. A reasonable maintenance plan is set up to develop the maximum network resilience under the conditions of available total restoration costs.

The network resilience is increased by means of adding new links before the disaster. Based on the calculation results in Table 6 , the network resilience increases by $22.6 \%$ by the most compared to 8.33 under the condition of adding S3 link and S5 link. But the network resilience performances under adding links of different combinations are slightly different between each other. The way of adding new links under sufficient funding conditions can be adopted to improve network resilience performance. Enhancing the reliability of links is the most direct and effective method to improve the road network resilience to resist seismic hazard.

7.3. Resilience Comparison. The resilience results obtained from the model proposed by this paper and Zhang have big differences presented in Table 5. The four reasons for appearing the above phenomena are described as follows. (1) Research object aspect: the research object of the Zhang model is the road network, including only bridges. However, this paper's research object is urban road networks, including urban bridges, tunnels, slopes, and ordinary road 


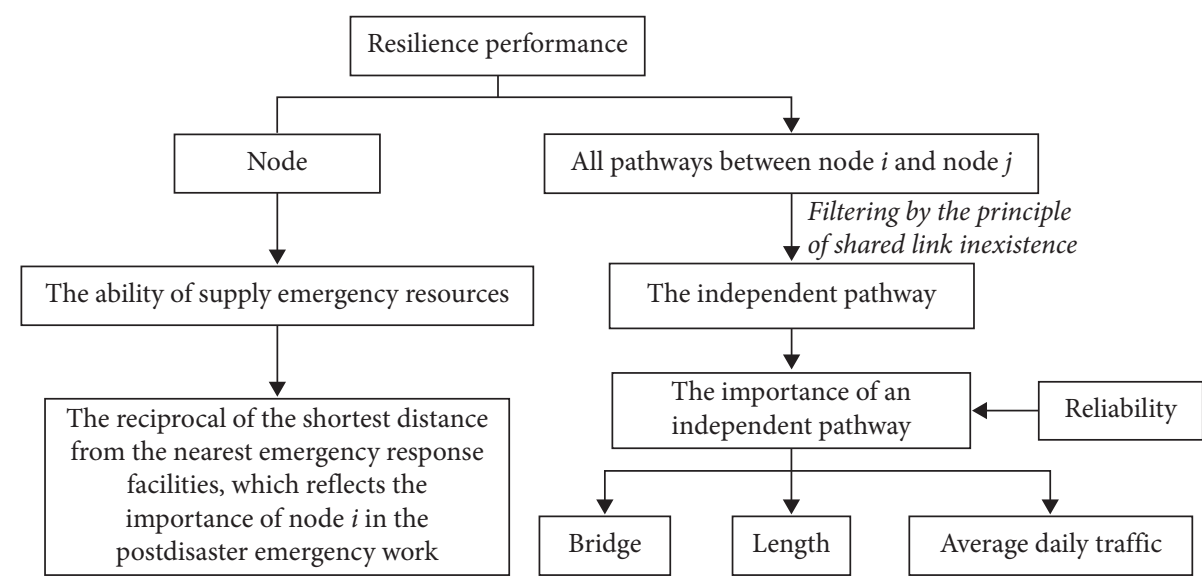

FIgure 2: The whole thought of Zhang model.

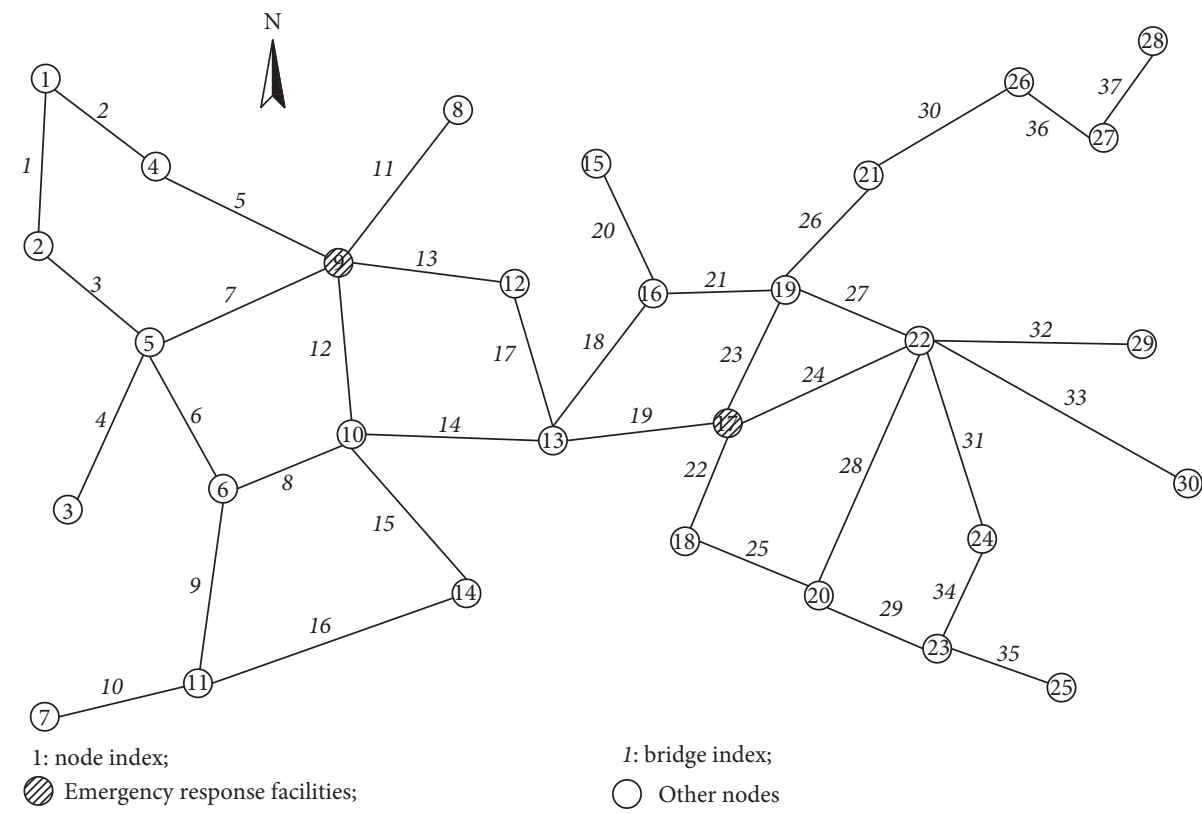

FIGURE 3: The diagram of the instance network.

TABLE 5: Resilience recovery of improving the reliability of the key links after the disaster.

\begin{tabular}{|c|c|c|c|c|c|}
\hline Serial number & Network state & $R P M$ & Increment percentage & Zhang model & Increment percentage \\
\hline 1 & $\begin{array}{l}\text { After disasters, that is the result in Section } 7.2 \\
\text { Using } 28 \text { unit costs, repairing a total of seven }\end{array}$ & 3.73 & - & 0.61 & - \\
\hline 2 & $\begin{array}{l}\text { links including links } 5,17,8,32,10,4 \text {, and } 33 \text {, and } \\
\text { making the reliability of seven links resume to } 0.999\end{array}$ & 4.36 & $16.9 \uparrow$ & 0.76 & $24.6 \uparrow$ \\
\hline 3 & $\begin{array}{l}\text { Using } 65 \text { unit costs, repairing a total of sixteen } \\
\text { links including links } 22,5,28,17,20,14,12,19,27 \text {, } \\
15,24,16,36,21,37 \text {, and } 31 \text {, and making the } \\
\text { reliability of sixteen links resume to } 0.999\end{array}$ & 5.67 & $52.0 \uparrow$ & 1.07 & $75.4 \uparrow$ \\
\hline 4 & $\begin{array}{l}\text { Using } 86 \text { unit costs, repairing a total of twenty-two } \\
\text { links including links } 27,11,19,16,10,31,5,17, \\
18,37,23,30,28,14,12,24,21,22,29,36,15, \\
\text { and } 20 \text {, and making the reliability of } \\
\text { twenty-two links resume to } 0.999\end{array}$ & 6.42 & $72.1 \uparrow$ & 1.24 & $103.3 \uparrow$ \\
\hline 5 & $\begin{array}{l}\text { Using } 105 \text { unit costs, repairing a total of } \\
\text { twenty-seven links including links } 7,6,11 \text {, } \\
\begin{array}{c}19,27,31,1,5,17,8,18,37,25,26,23,30, \\
28,14,2,24,21,35,22,29,36,20, \\
\text { and } 13 \text {, and making the reliability of } \\
\text { twenty-seven links resume to } 0.999\end{array}\end{array}$ & 7.15 & $91.7 \uparrow$ & 1.51 & $147.5 \uparrow$ \\
\hline
\end{tabular}


TABLE 6: Resilience recovery of changing the original network topology before the disaster.

\begin{tabular}{lccc}
\hline Serial number & Network state & $R P M$ & Increment percentage \\
\hline 1 & $\begin{array}{r}\text { The reliability of all links is 0.999 before disasters, } \\
\text { and network topology is still shown in Figure 3 }\end{array}$ & 8.33 & - \\
2 & Only adding S1 link and S4 link & 9.94 & $19.3 \uparrow$ \\
3 & Only adding S1 link and S5 link & 9.73 & $16.8 \uparrow$ \\
4 & Only adding S2 link and S4 link & 9.78 & $17.4 \uparrow$ \\
5 & Only adding S2 link and S5 link & 9.88 & $18.6 \uparrow$ \\
6 & Only adding S3 link and S4 link & 10.07 & $20.9 \uparrow$ \\
7 & Only adding S3 link and S5 link & 10.21 & $22.6 \uparrow$ \\
\hline
\end{tabular}

sections. When calculating the network's aseismic resilience, the link reliability calculation is more complicated due to the variety of links included in the urban road network. (2) Influence factor aspect: node importance of the Zhang model only takes into account the occupation degree of emergency resources. The model proposed by this paper in addition to considering resource occupies and considers the extent of the hub degree, center degree, and accessibility. The node status is assessed fully and comprehensively. For the pathway importance, the Zhang model only considers the length and average daily traffic of pathways. And the impact of travel time and the road width is not considered. The impact of road width is considered in this paper's established model. The types of road sections have also been extended on the basis of the Zhang model, including the tunnel, slope, and ordinary road section in addition to the bridge. (3) Analysis method aspect: Dijkstra's algorithm is used to obtain an independent pathway, and the algorithm coupled NSGA-II and Monte Carlo simulation is employed to determine the disaster reduction decisions in the Zhang model. The selfprepared MATLAB programs can finish the entire process calculation of aseismic resilience, and the programs have higher portability, editability, and computational efficiency. And the most important feature compared to other simulation methods is that the result aiming at a specific network is accurate and unique. (4) Calculation variable aspect: when changing the network topology as shown in Table 6, the hub degree, center degree, accessibility, and resources occupy degree of a node are changed with the variation of network topology, and the connecting type, amount, length, and reliability are changed at the same time. Therefore, the resilience performance needs to be calculated when the network topology changes. The above variations are considered to calculate the resilience repeatedly.

\section{Conclusions}

The research on the evaluation method of resilience performance for urban road networks facing an earthquake disaster is carried out in this study. Based on the urban road network's structural hierarchy and functional characteristics, the independent pathways without any shared links between nodes are the core of aseismic resilience performance. The aseismic resilience named $R P M$ of the overall network expresses the sum of the product of the node importance and the average number of reliable independent pathways. The hub degree, center degree, accessibility, and resources occupy degree are considered in node importance. Pathway importance is related to the average daily traffic, length, and travel time. The independent pathway's reliability is the continued production of the reliabilities of links included in this independent pathway. The resilience performance of the existing network, reconstructing network, and new network can be calculated by the established evaluation method of aseismic resilience, and the effects of all kinds of measures to enhance network resilience are evaluated in order to find the measures and methods of getting the maximum resilience performance under limited funding conditions. The resilience performances of the instance network under different states are calculated, and the results are compared. According to the evaluation model of aseismic resilience and analyses of instance networks, the means of enhancing resilience are as follows: (1) Improving the reliability of road sections to enhance network resilience, which is the most effective measure. (2) Adding new road sections to optimize the network topology, which indirectly affects the importance of nodes and pathways. (3) Changing the pattern of road traffic flow, including improving regulation speed or modifying traffic control regulations. (4) Reasonably siting of emergency response facility to improve the node important. The prepared MATLAB programs can achieve the efficient, accurate, and whole process calculation of the aseismic resilience, and the programs have the characteristics of open resources, reusability, and portability.

\section{Data Availability}

The result data used to support the findings of this study are included within the supplementary information file.

\section{Conflicts of Interest}

The authors declare that they have no conflicts of interest.

\section{Acknowledgments}

This research was funded by the Chongqing Natural Science Foundation (CSTC2019jcyj-msxmX0781), the Youth Project of Science and Technology Research Program of Chongqing Education Commission of China (KJQN201901332), and the National Natural Science Foundation of China (51678544). 


\section{Supplementary Materials}

In the supplemental file of this paper, Table $\mathrm{S} 1$ lists the data of reliability, average daily traffic, and restoration cost for each road section in the network of Figure 3. Table S2 calculates node importance in the emergency recovery phase by using the model proposed in this paper. Figure S1 shows the results of Table S2 graphically. Table S3 takes the independent pathway calculation of node 1 to other 29 nodes as an example. Node connection sequences of pathways, ADT, ADT index, total length, length index, pathway importance, and pathway reliability are given in Table S3. (Supplementary Materials)

\section{References}

[1] K. Rus, V. Kilar, and D. Koren, "Resilience assessment of complex urban systems to natural disasters: a new literature review," International Journal of Disaster Risk Reduction, vol. 31, pp. 311-330, 2018.

[2] Y. Yang, S. T. Ng, F. J. Xu, and M. Skitmore, "Towards sustainable and resilient high density cities through better integration of infrastructure networks," Sustainable Cities and Society, vol. 42, pp. 407-422, 2018.

[3] L. Wei and S. Zhaoyang, "Review of studies on the resilience of urban critical infrastructure networks," Reliability Engineering \& System Safety, vol. 2019, pp. 1-10, 2019.

[4] Z. Yaoming, W. Junwei, and Y. Hai, "Resilience of transportation systems: concepts and comprehensive review," IEEE Transactions on Intelligent Transportation Systems, vol. 20, pp. 1-15, 2019.

[5] Y.-J. Lee, J. Song, P. Gardoni, and H.-W. Lim, "Post-hazard flow capacity of bridge transportation network considering structural deterioration of bridges," Structure \& Infrastructure Engineering, vol. 7, no. 7-8, pp. 509-521, 2011.

[6] S. Guikema and P. Gardoni, "Reliability estimation for networks of reinforced concrete bridges," Journal of Infrastructure Systems, vol. 15, no. 2, pp. 61-69, 2009.

[7] P. Bocchini and D. M. Frangopol, "Connectivity-based optimal scheduling for maintenance of bridge networks," Journal of Engineering Mechanics, vol. 139, no. 6, pp. 760-769, 2013.

[8] A. Chen, J. Kim, Z. Zhou, and P. Chootinan, "Alpha reliable network design problem," Transportation Research Record: Journal of the Transportation Research Board, vol. 2029, no. 1, pp. 49-57, 2007.

[9] Y.-S. Qian, M. Wang, H.-X. Kang, J.-W. Zeng, and Y.-F. Liu, "Study on the road network connectivity reliability of valley city based on complex network," Mathematical Problems in Engineering, vol. 2012, Article ID 430785, 14 pages, 2012.

[10] S. Peeta, F. Sibel Salman, D. Gunnec, and K. Viswanath, "Predisaster investment decisions for strengthening a highway network," Computers \& Operations Research, vol. 37, no. 10, pp. 1708-1719, 2010.

[11] E. K. Morlok and D. J. Chang, "Measuring capacity flexibility of a transportation system," Transportation Research Part A: Policy and Practice, vol. 38, no. 6, pp. 405-420, 2004.

[12] S. E. Chang and N. Nojima, "Measuring post-disaster transportation system performance: the 1995 Kobe earthquake in comparative perspective," Transportation Research Part A: Policy and Practice, vol. 35, no. 6, pp. 475-494, 2001.
[13] W. H. Ip and D. Wang, "Resilience and friability of transportation networks: evaluation, analysis and optimization," IEEE Systems Journal, vol. 5, no. 2, pp. 189-198, 2011.

[14] W.-L. Shang, Y. Chen, C. Song, and W. Y. Ochieng, "Robustness analysis of urban road networks from topological and operational perspectives," Mathematical Problems in Engineering, vol. 2020, Article ID 5875803, 12 pages, 2020.

[15] W. Zhang and N. Wang, "Resilience-based risk mitigation for road networks," Structural Safety, vol. 62, pp. 57-65, 2016.

[16] W. Zhang, N. Wang, and C. Nicholson, "Resilience-based post-disaster recovery strategies for road-bridge networks," Structure and Infrastructure Engineering, vol. 13, no. 11, pp. 1404-1413, 2017.

[17] W. Zhang, N. Wang, C. Nicholson, and M. H. Tehrani, “A stage-wise decision framework for transportation network resilience planning," Physics, vol. 2018, pp. 1-27, 2018.

[18] J. Wang, H. Liu, and J. Zhu, "Study on road network recovery under snow and ice conditions based on the perspective of resilient city," Chinese Journal of Management Science, vol. 26, pp. 177-187, 2018.

[19] Z. Li, C. Jin, P. Hu, and C. Wang, "Resilience-based transportation network recovery strategy during emergency recovery phase under uncertainty," Reliability Engineering \& System Safety, vol. 188, pp. 503-514, 2019.

[20] D.-m. Zhang, F. Du, H. Huang, F. Zhang, B. M. Ayyub, and M. Beer, "Resiliency assessment of urban rail transit networks: shanghai metro as an example," Safety Science, vol. 106, pp. 230-243, 2018.

[21] D. Forcellini, "A new methodology to assess indirect losses in bridges subjected to multiple hazards," Emerging Science Journal, vol. 4, no. 1, pp. 4-10, 2019.

[22] J. Wang and H. Liu, "Snow removal resource location and allocation optimization for urban road network recovery: a resilience perspective," Journal of Ambient Intelligence and Humanized Computing, vol. 10, no. 1, pp. 395-408, 2019.

[23] Y. Yang, S. T. Ng, S. Zhou, F. J. Xu, and H. Li, "A physicsbased framework for analyzing the resilience of interdependent civil infrastructure systems: a climatic extreme event case in Hong Kong," Sustainable Cities and Society, vol. 47, pp. 1-14, 2019.

[24] Q. Zou and S. Chen, "Enhancing resilience of interdependent traffic-electric power system," Reliability Engineering and System Safety, vol. 191, pp. 1-18, 2019.

[25] S. Kameshwar, D. T. Cox, A. R. Barbosa et al., "Probabilistic decision-support framework for community resilience: incorporating multi-hazards, infrastructure interdependencies, and resilience goals in a Bayesian network," Reliability Engineering and System Safety, vol. 191, pp. 1-21, 2019.

[26] Y. G. Dmitriev and G. M. Koshkin, "Influence of using additional information on the accuracy of estimation of the reliability function characteristics at a finite size of observations," Russian Physics Journal, vol. 62, no. 4, pp. 649-657, 2019.

[27] E. D. Guo, Z. Zhao, Z. R. Wang, Z. Liu, L. Gao, and Y. D. Wang, "Improved methodology for seismic estimation of highway beam bridges," Journal of Beijing University of Technology, vol. 39, no. 2, pp. 192-197, 2013.

[28] R. Q. Lan, X. J. Li, B. Feng, and Y. S. Wang, "Study on the seismic damage evaluation method of urban bridges under the near-field strong ground motion," Journal of Natural Disasters, vol. 26, no. 4, pp. 135-142, 2017.

[29] H. Sun and H. Y. Gao, "Study on seismic damage prediction method for urban bridges based on PSO-PPE," World Earthquake Engineering, vol. 33, no. 3, pp. 152-159, 2017. 
[30] J. Q. Lin, X. Q. Fang, and J. L. Liu, "Earthquake damage evaluation of tunnels," Applied Mechanics and Materials, vol. 170-173, pp. 1402-1409, 2012.

[31] Y. Q. Wang, H. B. Xin, Y. P. Gao, and G. S. Zhou, "Study on comprehensive index method for predicting earthquake-induced landslides," Chinese Journal of Geotechnical Engineering, vol. 23, no. 3, pp. 311-314, 2001.

[32] P. Du, "Improvement for the calculating method of debris piling problem in the earthquake damage forecasting of the transportation system," World Earthquake Engineering, vol. 23, no. 1, pp. 161-164, 2007. 